\title{
Yapay Sinir Ağları İle Çeyrek Daire Yarıklı Mikroşerit Yama Antenin Rezonans Frekansının Belirlenmesi
}

\author{
Muhammed Can Bayram ${ }^{1}$, Sueda Güzelbakan ${ }^{2}$, Esin Karpat ${ }^{3 *}$ \\ ${ }^{1}$ Bursa Uludağ Üniversitesi, Mühendislik Fakültesi, Elektrik Elektronik Mühendisliği Bölümü, Bursa, Türkiye, (ORCID: 0000-0003-1140-2980), \\ m.canbayram16@gmail.com \\ 2 Bursa Uludağ Üniversitesi, Mühendislik Fakültesi, Elektrik Elektronik Mühendisliği Bölümü, Bursa, Türkiye (ORCID: 0000-0002-6052-2295), \\ suedaguzelbakan@uludag.edu.tr \\ 3* Bursa Uludağ Üniversitesi, Mühendislik Fakültesi, Elektrik Elektronik Mühendisliği Bölümü, Bursa, Türkiye (ORCID: 0000-0002-2740-8183), \\ esinoz@uludag.edu.tr
}

(International Conference on Design, Research and Development (RDCONF) 2021 - 15-18 December 2021)

(DOI: 10.31590/ejosat.1039855)

ATIF/REFERENCE: Bayram M.C, Güzelbakan S. \& Karpat E. (2021). Yapay Sinir Ağları İle Çeyrek Daire Yarıklı Mikroşerit Yama Antenin Rezonans Frekansının Belirlenmesi. Avrupa Bilim ve Teknoloji Dergisi, (32), 716-720.

$\ddot{\mathbf{O} z}$

Geliştirilen yeni mikroşerit anten geometrilerinde çalışma frekansını doğrudan hesaplayabilecek analitik modeller bulunmadığından çok katmanlı ağ yapısına dayanan yapay sinir ağı (YSA) modelleri çalışma frekansını belirlemek için yaygın olarak kullanılmaktadır. $\mathrm{Bu}$ çalışmada çeyrek daire yarıklı mikroșerit antene ait farklı giriş verileri (anten fiziksel parametreleri) için rezonans frekansını elde edilmesini sağlayan çok katmanlı YSA modeli oluşturulmuştur. Bu veriler, antenin rezonans frekansı 1,475-2,45 $\mathrm{GHz}$ aralığında olacak şekilde belirlenmiştir. ANSYS HFSS benzetim programı kullanılarak elde edilen 120 farklı anten verisi YSA modelinde eğitim ve test süreçlerinde kullanılmıştır. Geliştirilen YSA modeli ile antenin farklı fiziksel parametreleri için rezonans frekansı elde edilmiştir. Benzetim ve test sonuçları karşılaştırıldığında 40 test verisi için ortalama hata değerinin $\% 0,34$ olduğu görülmektedir. Bu sonuçlara göre rezonans frekansının belirlenmesi için çok katmanlı ağ yapısının başarılı bir şekilde kullanılabileceği gösterilmiştir. YSA modelinin oluşturulmasında sınıflandırıcı performans algoritmaları MATLAB yazılımı kullanılarak geliştirilmiş̧ir.

Anahtar Kelimeler: Rezonans Frekansı Yapay Sinir Ağları, Mikroşerit Anten, Anten Tasarımı.

\section{Resonance Frequency Determination of Quarter Circular Slotted Microstrip Patch Antenna using Artificial Neural Networks}

\begin{abstract}
Since there are no analytical models that can directly calculate the operating frequency in the newly developed microstrip antenna geometries, artificial neural network (ANN) models based on the multilayer network structure are widely used to obtain the operation frequency. A multilayer ANN model is developed to determine the resonance frequency of a quarter-circular slotted microstrip antenna for various input data (antenna parameters). These data are varied to obtain the resonance frequency of the antenna in the range of 1.475-2.45 GHz. 120 different antenna data obtained by the ANSYS HFSS simulation program are used in the training and testing processes of the ANN model. The resonance frequency of the antenna is determined using the created ANN model for various parameters of the antenna. When the simulation and test results are compared, a mean error value for 40 test data is $0.34 \%$ is obtained. The results show that the multilayer network structure can be successfully used to determine the resonance frequency. The ANN model is created using MATLAB software to construct classifier performance algorithms.
\end{abstract}

Keywords: Resonance Frequency, Artificial Neural Networks, Microstrip Antenna, Antenna Design

*Sorumlu Yazar: esinoz@uludag.edu.tr 


\section{Giriş}

Anten, elektromanyetik enerjiyi çok yönlü olarak istenilen yönlerde yaymak için kullanılan bir cihazdır (Balanis, 2016). Mikroşerit antenler geleneksel şekilli antenler ile kıyaslandığında düşük maliyet, mikrodalga devrelerine kolay entegre edilebilme, hacim ve boyutlarının küçük olması gibi birçok avantajlı duruma da sahiptir (Garg vd., 2001). Bu avantajlar göz önünde bulundurulduğunda mikroşerit antenlerin radar sistemleri (Rajan \& Vivek, 2019), uzay araçları (Jyosthna vd., 2020), kablosuz iletişim (Sainati, 1996) gibi birçok mikrodalga uygulama alanlarında kullanıldığ gözlemlenmektedir. Mikroşerit antenler ile ilgili ilk fikir Deschamp tarafından 1953 'te sunulmuştur. Anten çalışmalarının hız kazanması ise 1970'li yıllara dayanmaktadır. Mikroşerit yama anten temel olarak üç ana bölümden oluşmaktadır. Yer düzlemi, iletken bir yama ve bu iki bölge arasında kalan dielektrik malzemeden oluşmuş alttaş temel mikroşerit yama anteni tanımlamaktadır. (Kumar \& Ray, 2003). Mikroşerit antenler için yaygın olarak kare, üçgen, daire vb. geometrileri kullanılmaktadır (Dearnley \& Barel, 1989; Tang vd., 1997; Nayna vd., 2014; Chew vd., 2008; Kayabaş1 vd., 2013). Doğrusal olmayan bir problem olarak adlandırılan mikroşerit antenlerin çalışma frekansının belirlenmesinin analitik olarak çözümü oldukça zordur. Yaygın geometrilerin istenilen frekansları karşılamadığ 1 durumlarda mevcut anten geometrileri değiştirilerek yeni anten geometrileri oluşturulmaktadır (Singh vd., 2016; Prakasam \& Reddy, 2021; İmamoğlu, 2019). Geliştirilen yeni geometrilerin çalışma frekansını doğrudan hesaplayabilecek analitik modeller bulunmamaktadır.

Çok katmanlı ağ yapısına dayanan YSA modelleri (Haykin, 2004), mikroşerit antenlerin çalışma frekansını belirlemek için yaygın olarak kullanılmaktadır. Hızlı öğrenme ve modelleme yapabilme yeteneği sayesinde YSA ile hizlı hesaplamalar yap1labilmektedir. $\mathrm{Bu}$ yöntem, elektromanyetik problemlerin çözümünde oldukça tercih edilmektedir (Oluyemi vd., 2021; Zhang vd., 2021; Agatonović vd., 2011; Sanchez, 2004).

Mikroşerit anten yapılarının rezonans frekansının belirlenmesinde YSAların ilk uygulamalaları kare (Mishra \& Patnaik, 1998), dikdörtgen (Türker vd., 2007) ve dairesel (Sağıroğlu vd., 1998) vb. standart geometriler için gerçekleştirilmiştir. Hızlı ögrenme ve modelleme yapabilme yeteneği sayesinde YSA, farklı geometrilere sahip yama anten uygulamalarında da kullanılabilmektedir. A. Kayabaşı ve arkadaşları ise rezonans frekansı UHF bandında yer alan $\mathrm{H}$ geometriye sahip kompakt mikroşerit anteni (HKMA) çok katmanlı algılayıcılara (ÇKA) dayanan YSA ile tasarlamışlardır (Kayabaşı, Biçer, Akdağlı, \& Toktaş, 2013).

$\mathrm{Bu}$ çalışmada, 1,475-2,45 GHz frekans aralığı için tasarlanan mikroşerit antene ait (İmamoğlu, 2019) farklı giriş verileri için YSA modeli oluşturulmuştur. Geliştirilen YSA modeli kullanılarak anten fiziksel boyutlarının değişimine bağlı olarak çalışma frekansı belirlenmiştir. Elde edilen sonuçlar benzetim verileri ile karşılaştırılmıştır.

\section{Materyal ve Yöntem}

\subsection{Mikroșerit Anten Tasarımı}

Şekil 1'de dış boyutları L,W ve oyuk yarıçapı r, alttaş kalınlığ 1 ise h olarak belirlenen anten geometrisi görülmektedir (İmamoğlu, 2019). Altı farklı frekans değeri için antenin yama boyutları dikdörtgen mikroşerit yama antene ait denklemler kullanılarak hesaplanmıştır (Balanis, 2016). Şekil 1'de görüldüğü gibi dikdörtgen yama anteninin köşelerinden yarıçapı $r$ olan çeyrek daire yarıkları çıkarılarak tasarım gerçekleştirilmiştir. Beş farklı alttaş kalınlığı (h) için yarıçap değerleri $0,1 \mathrm{~L}$ ile $0,5 \mathrm{~L}$ aralığında $0,1 \mathrm{~L}$ kadar artırılarak benzetimler gerçekleştirilmişitr ve farklı $(\mathrm{r}, \mathrm{h})$ değerlerine karşılık gelen rezonans frekansı değerleri elde edilmiştir. Antenin benzetimleri, Sonlu Elemanlar Metodu (FEM) tabanlı ANSYS HFSS (yüksek frekanslı yapısal benzetim) programı ile sayısal olarak gerçekleştirilmiştir. Fiziksel parametre değişimlerinin rezonans frekansına etkisi incelenmiştir. Benzetimleri gerçekleştirilen antenlerin boyutları Tablo 1'de yer almaktadır.

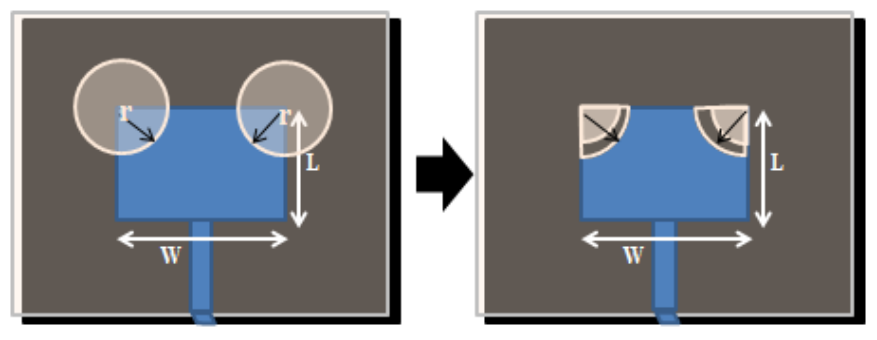

Şekil 1. Mikroşerit Anten Geometrisi

Tablo 1. Mikroşerit Anten Boyutları ve $(r, h)$ Değerlerinin Değişimi

\begin{tabular}{|c|c|c|c|c|c|c|}
\hline \multirow{2}{*}{$\begin{array}{c}\mathbf{f}_{\mathbf{r}} \\
(\mathbf{G H z})\end{array}$} & \multicolumn{2}{|c|}{$\begin{array}{l}\text { MIKROŞERIT } \\
\text { ANTEN DIŞ } \\
\text { BOYUTLARI }\end{array}$} & \multicolumn{4}{|c|}{ YARIK KESME BOYUTLARI } \\
\hline & $\mathbf{L}(\mathbf{m m})$ & $\mathbf{W}(\mathbf{m m})$ & Değişken & Min & Max & $\Delta$ \\
\hline \multirow{2}{*}{1,43} & \multirow{2}{*}{79,02} & \multirow{2}{*}{57,14} & $\mathbf{r}$ & $0,1 \mathrm{~L}$ & $0,5 \mathrm{~L}$ & $0,1 \mathrm{~L}$ \\
\hline & & & $\mathbf{h}$ & $0,6 \mathrm{~h}_{\max }$ & $1 \mathrm{~h}_{\text {max }}$ & $0,1 \mathrm{~h}_{\max }$ \\
\hline \multirow{2}{*}{1,63} & \multirow{2}{*}{69,72} & \multirow{2}{*}{50,42} & $\mathbf{r}$ & $0,1 \mathrm{~L}$ & $0,5 \mathrm{~L}$ & $0,1 \mathrm{~L}$ \\
\hline & & & $\mathbf{h}$ & $0,6 \mathrm{~h}_{\max }$ & $1 \mathrm{~h}_{\max }$ & $0,1 \mathrm{~h}_{\max }$ \\
\hline \multirow{2}{*}{1,83} & \multirow{2}{*}{62,38} & \multirow{2}{*}{45,11} & $\mathbf{r}$ & $0,1 \mathrm{~L}$ & $0,5 \mathrm{~L}$ & $0,1 \mathrm{~L}$ \\
\hline & & & $\mathbf{h}$ & $0,6 \mathrm{~h}_{\max }$ & $1 \mathrm{~h}_{\max }$ & $0,1 \mathrm{~h}_{\max }$ \\
\hline \multirow{2}{*}{2,03} & \multirow{2}{*}{56,44} & \multirow{2}{*}{40,82} & $\mathbf{r}$ & $0,1 \mathrm{~L}$ & $0,5 \mathrm{~L}$ & $0,1 \mathrm{~L}$ \\
\hline & & & $\mathbf{h}$ & $0,6 \mathrm{~h}_{\max }$ & $1 \mathrm{~h}_{\max }$ & $0,1 \mathrm{~h}_{\max }$ \\
\hline \multirow{2}{*}{2,25} & \multirow{2}{*}{51,53} & \multirow{2}{*}{37,27} & $\mathbf{r}$ & $0,1 \mathrm{~L}$ & $0,5 \mathrm{~L}$ & $0,1 \mathrm{~L}$ \\
\hline & & & $\mathbf{h}$ & $0,6 \mathrm{~h}_{\max }$ & $1 \mathrm{~h}_{\max }$ & $0,1 \mathrm{~h}_{\max }$ \\
\hline \multirow{2}{*}{2,43} & \multirow{2}{*}{47,41} & \multirow{2}{*}{34,28} & $\mathbf{r}$ & $0,1 \mathrm{~L}$ & $0,5 \mathrm{~L}$ & $0,1 \mathrm{~L}$ \\
\hline & & & $\mathbf{h}$ & $0,7 \mathrm{~h}_{\max }$ & $1 \mathrm{~h}_{\max }$ & $0,1 \mathrm{~h}_{\max }$ \\
\hline
\end{tabular}




\subsection{YSA ile Mikroşerit Antenin Çalışma Frekansının Hesaplanması}

Elde ettiği veriler ile olaylar arasındaki ilişkileri öğrenerek sonrasında hiç öğrenmediği örnekler üzerinde öğrendiği bilgileri kullanarak karar veren sistemler YSA olarak adlandırılır (Haykin, 2004). Sinapslar yardımı ile iletişim kuran biyolojik sinir hücreleri biyolojik sinir sistemini oluşturur. Biyolojik sinir sisteminin bu prensibinden yola çıkılarak YSA modelleri geliştirilmişstir. Sinir hücreleri işledikleri bilgilerin axonları yolu ile hücrelere iletimini sağlar. Yapay sinir hücreleri ise benzer şekilde dışarıdan alınan bilgileri ilk olarak bir toplama fonksiyonundan geçirerek toplar ve ardından aktivasyon fonksiyonundan geçirerek çıktının üretilip ağ bağlantıları üzerinden diğer hücrelere yani proses elemanlara aktarımını sağlar. Farklı tiplerde toplama ve aktivasyon fonksiyonları mevcuttur. Ağırlık değerleri olarak yapay sinir ağlarını birbirlerine bağlayan bağlantı değerleri alınmaktadır. Üç katman halinde bir araya gelerek birbirlerine paralel olarak proses elemanları bir ağ oluştururlar.

YSA işleminin temelini oluşturan bilgi işleme birimi bir nöron ya da bir yapay sinir hücresi olarak tanımlanabilir. (Haykin, 1994). Bir nöronun çıktısı Denklem 1'deki gibi formüle edilebilir:

$$
y_{i}=f\left(\sum w_{j i} x_{i}\right)
$$

Denklem 1'de verilen $y_{i}$ değeri çıkışı, $x_{i}$ değeri giriş verilerini, $\mathrm{w}_{\mathrm{ji}}$ gizli katman nöron ağırlığını ifade etmektedir. $f$ fonksiyonu $\mathrm{x}_{\mathrm{i}}$ değeri ile $\mathrm{w}_{\mathrm{ji}}$ nin çarpılarak toplanması sonucunda elde edilir. Burada $f$, hiperbolik tanjant eşik fonksiyonu olarak kullanılmıştır. Farklı eğitim algoritmalarını kullanıp ağın ağırlıklarında (w) optimizasyon yaparak bir ağı eğitme işlemi gerçekleştirilir. Çok Katmanlı Sinir Ağ eğitiminde, hızlı bir optimizasyon algoritması olan ve mühendislik uygulamalarında oldukça sık kullanım alanına sahip Levenberg-Marguart (LM) algoritması tercih edilmiştir (Ataş vd., 2013).

\subsubsection{YSA Modelinin Eğitimi}

Geliştirilen YSA modeli Şekil 2'de görülmektedir. Öğrenme algoritması olarak LM, gizli katmanda hiperbolik tanjant sigmoid fonksiyonu ve çıkış katmanında transfer fonksiyonu olarak saf doğrusal fonksiyon kullanılmıştır. İstenilen çıkış yanıtlarına ulaşmak için giriş katmanına gönderilen veriler eğitilirken gizli katmandaki sinaptik ağırlıklar değiştirilmiştir. 100 epok sayısı ile eğitim hatası minimuma indirgendiğinden eğitim işlemi sonlandırılmıştır. Sonuç olarak eğitim boyunca görmediği test veri seti ile eğitilmiş sinir ağının, doğruluğu ölçülmüştür. ANSYS HFSS benzetim programı kullanılarak gerçekleştirilen 120 farklı anten tasarımı (İmamoğlu, 2019) içinden 80 veri seti eğitim süreci için, eğitime katılmayan 40 veri seti ise test sürecinde kullanılmıştır

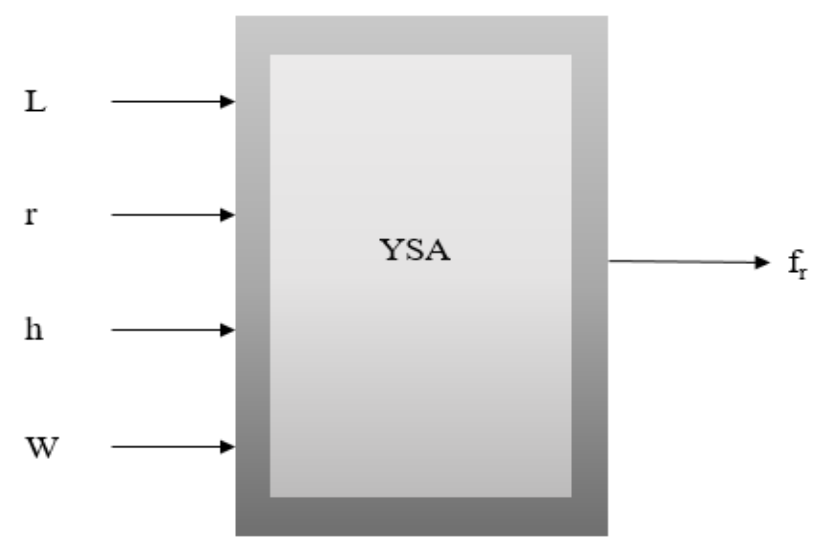

Şekil 2. YSA Eğitim Süreci

\subsubsection{YSA Modeli Test Süreci}

Çok katmanlı algılayıcılara dayanan YSA modeli, rezonans frekansını hesaplayabilmek için kullanılmıştır. Bu çalışmada, üç katmandan oluşan bir YSA modeli tercih edilmiştir. Katman sayısı üçten daha fazla olduğunda Üç katmandan daha büyük mimarilerde karmaşıklık artmaktadır. Bu nedenle tek gizli katman mimarisi en basit mimari olarak seçilmiştir.

ÇKA tasarımında giriş katmanındaki nöron sayısını, kullanılan geometrik (uzunluk, genişlik, yarıçap, alttaş kalınlığı) parametre sayısı, çıkış katmanındaki nöron sayısını ise rezonans frekans sayısını belirtmektedir.

YSA modelinde giriş parametreleri $x_{1}, x_{2}, x_{3}$ ve $x_{4}$ olarak belirlenmiştir. Çıkış parametresi ise $y^{\prime}$ dir. Gerçekleştirilen denemeler sonucunda; giriş katmanı dört nörondan, gizli katmanı 10 nörondan ve çıkış katmanı da 1 nörondan oluşturulmuş olan bir YSA topolojisinin en uygun ağ modeli olacağı belirlenmiştir. Önerilen YSA modeli Şekil 3 'te gösterilmiştir. Oluşturulan YSA modelindeki girişler anten yama boyutları (L,W), alttaş kalınlığı (h) ve çeyrek daire yarık yarıçapı (r) olarak alınmıştır. Çıkışta elde edilen parametre rezonans frekansidir.

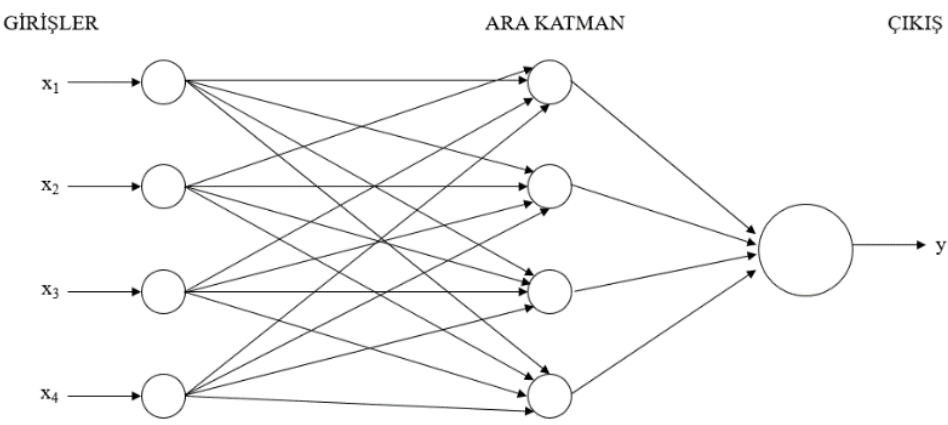

Şekil 3. YSA Modeli 


\section{Rezonans Frekansının YSA ile Hesaplanması}

ÇKA eğitiminde, benzetimi gerçekleștirilen 120 antenin rezonans frekansı değerlerinin 80’i eğitim işleminde kullanılmıştır. 40 benzetim verisi ise daha önce eğitime katılmamış ve ağın doğruluğunun test edilmesi sürecinde bu verilerden yararlanılmıştır. Şekil 4'te tasarlanan ağ modeli gösterilmiştir. LM algoritması ile eğitilen ÇKA ağı için epok sayısı 100 olarak belirlenmiştir. ÇKA'nın başarı sonucu, test verileri kullanilarak YSA modeli ile elde edilen rezonans frekansı değerleri ile aynı test verileri kullanılarak oluşturulan anten modelinin HFSS benzetimi sonucunda elde edilen rezonans frekansı değerleri Tablo 2'de verilmiştir. Benzetim sonuçları ile test sonuçları karşılaştırıldığında 40 test verisi için \% 0.34 ortalama hata değeri elde edilmiştir. Bu sonuçlara göre, rezonans frekansının belirlenmesi için ÇKA'nın başarılı bir şekilde kullanılabileceği gösterilmiştir. Sonuç olarak eğitilen bu ağ girişlerine farklı anten fiziksel parametreleri verilerek karşılık gelen rezonans frekansı değeri hesaplanabilmektedir.

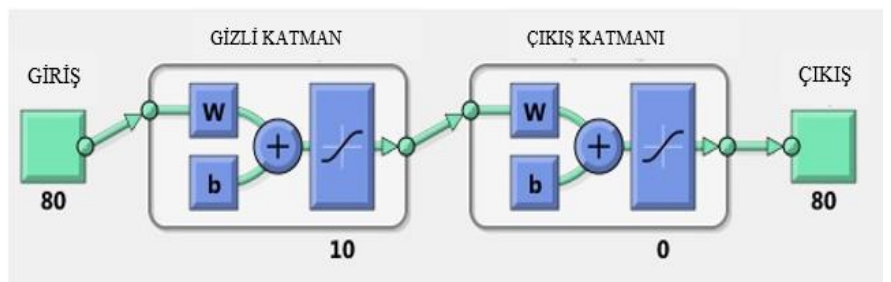

Şekil 4. Tasarlanan A $\breve{g}$ Modeli

\section{Sonuç}

$\mathrm{Bu}$ çalışmada, mikroşerit antenin rezonans frekansının belirlenmesinde ÇKA yapısını kullanan YSA modeli önerilmiștir. Elektromanyetik sayısal hesaplama programı olan ANSYS HFSS ile 120 adet mikroşerit antenin rezonans frekansını belirleyebilmek için benzetim gerçekleştirilmiştir. Önerilen YSA modeli için eğitim verisi olarak 80 anten verisi kullanılırken, test için kalan 40 anten verisi kullanılmıştır. Eğitim ve test verileri için hesaplanan değerlerin, YSA ve benzetim sonuçları ile iyi bir uyum içerisinde olduğu görülmüştür. Bu sonuçlara göre Çok Katmanlı A ̆g yapısına dayanan YSA'nın başarılı bir şekilde mikroşerit antenlerin rezonans frekansının belirlenmesinde kullanılabileceği gösterilmiştir. YSA modeli kolay uygulama yöntemleri ve elde edilen sonuçların doğruluğu açısından diğer uygulamalar ile karşılaştırıldığında avantajlı olduğu görülmektedir.

Tablo 2. Test Verileri için YSA ile Elde Edilen Rezonans Frekansları ile Benzetim Sonuçlarının Karşılaştırılması

\begin{tabular}{|c|c|c|c|c|c|c|}
\hline $\mathbf{W}(\mathbf{m m})$ & $\mathbf{L}(\mathbf{m m})$ & $r(\mathbf{m m})$ & $\mathbf{h}(\mathbf{m m})$ & $\begin{array}{l}f_{r}(\mathrm{GHz}) \\
\text { Benzetim }\end{array}$ & $\begin{array}{c}\text { fr }(\mathrm{GHz}) \\
\text { YSA } \\
\end{array}$ & $\begin{array}{c}\text { Yüzde Hata } \\
\%\end{array}$ \\
\hline 47,41 & 34,28 & 3,43 & 4,80 & 2,50 & 2,50 & 0,015 \\
\hline 79,02 & 57,14 & 22,86 & 9,00 & 1,51 & 1,53 & 1,27 \\
\hline 79,02 & 57,14 & 17,14 & 7,00 & 1,56 & 1,56 & 0,19 \\
\hline 69,72 & 50,42 & 25,21 & 7,94 & 1,74 & 1,75 & 0,20 \\
\hline 69,72 & 50,42 & 15,13 & 6,18 & 1,78 & 1,77 & 0,40 \\
\hline 62,38 & 45,11 & 13,54 & 5,53 & 2,00 & 1,99 & 0,49 \\
\hline 56,44 & 40,82 & 4,08 & 5,00 & 2,13 & 2,13 & 0,15 \\
\hline 51,53 & 37,27 & 14,91 & 5,22 & 2,41 & 2,41 & 0,17 \\
\hline 47,41 & 34,28 & 3,43 & 5,40 & 2,47 & 2,46 & 0,22 \\
\hline 47,41 & 34,28 & 10,29 & 4,20 & 2,62 & 2,62 & 0,30 \\
\hline 47,41 & 34,28 & 3,43 & 4,80 & 2,50 & 2,50 & 0,015 \\
\hline \multicolumn{6}{|c|}{ Ortalama Yüzde Hata } & 0,34 \\
\hline
\end{tabular}




\section{Kaynakça}

Agatonović, M., Marinković, Z., \& Marković, V. (2011). Modeling of Microwave Pyramidal Absorbers using Artificial Neural Networks. 2011 19thTelecommunications Forum (TELFOR) Proceedings of Papers, 1012-1015.

Ataş, İ., Kurt, M. B., \& Ataş, M. (2013). Açıklık Kuplajlı Mikroşerit Yama Antenler İçin Yapay Sinir A ̆g Modeli. Dicle Üniversitesi Mühendislik Fakültesi Mühendislik Dergisi , 69-75.

Balanis, C. A. (2016). Antenna Theory: Analysis and Design, 4th Edition. John Wiley \& Sons, Inc.

Chew, W. C., Kong, J. A., \& Shen, L. C. (2008). Radiation characteristics of a circular microstrip antenna. Journal of Applied Physics 51. doi:10.1063/1.328138

Dearnley, R. W., \& Barel, A. R. (1989). A Comparison Of Models To Determine The Resonant Frequencies Of A Rectangular Microstrip Antenna. IEEE Transactions on Antennas and Propagation vol. 37, 114-118. doi:10.1109/8.192173

Garg, R., Bharrtia, P., Bahl, I., \& Ittipiboon, A. (2001). Microstrip Antenna Design Handbook. London: Artech House.

Haykin, S. (2004). Neural Networks A Comprehensive Foundation. Indian: Pearson Education Singapore Pte Ltd.

İmamoğlu, F. (2019). Yüksek Lisans Tezi. Mikroşerit Antenlerin Tasarım Ve Performans Optimizasyonu. Bursa, Türkiye: Bursa Uludağ Üniversitesi, Fen Bilimleri Enstitüsü.

Jyosthna, R., Sunny, R. A., Jugale, A. A., \& Ahmed, M. R. (2020). Microstrip Patch Antenna Design for Space Applications . 2020 International Conference on Communication and Signal Processing (ICCSP), (s. 406410). "Microstrip Patch Antenna Design for Space Applications. doi:10.1109/ICCSP48568.2020.9182250.

Kayabaşı, A., Biçer, M., Akdağlı, A., \& Toktaş, A. (2013). Uhf Bandında Çalışan H Şekilli Kompakt Mikroşerit Antenlerin Rezonans Frekansının Yapay Sinir Ağları Kullanarak Hesaplanmas1. Gazi Üniversitesi Mühendislik Mimarlık Fakültesi Dergisi 26 (4), 833-840.

Kumar, G., \& Ray, K. P. (2003). Broadband Microstrip Antennas, Artech House, USA, 2003. USA: Artech House.

Mishra, R., \& Patnaik, A. (1998). Neural Network-Based CAD Model For The Design Of Square-Patch Antennas. IEEE Transactions on Antennas and Propagation, vol. 46, no. 12, 1890-1891. doi:10.1109/8.743842.

Nayna, T. F., Baki, A. K., \& Ahmed, F. (2014). Comparative Study Of Rectangular And Circular Microstrip Patch Antennas İn X Band. 2014 International Conference on Electrical Engineering and Information \& Communication Technology. Dhaka, Bangladesh.

Oluyemi, O., Laforge, P., \& Bais, A. (2021). Artificial Neural Network for Microwave Filter Design using a Circuit Model. 2021 IEEE 19th International Symposium on Antenna Technology and Applied Electromagnetics (ANTEM) (s. 1-2). Winnipeg, MB, Canada: IEEE. doi: 10.1109/ANTEM51107.2021.9518641

Prakasam, V., \& Reddy, N. (2021). Hexagonal Shaped Microstrip Patch Antenna Design for $2.45 \mathrm{GHz}$ WLAN System. Proceedings of the Sixth International Conference on Inventive Computation Technologies [ICICT 2021]. Coimbatore, India. doi:10.1109/ICICT50816.2021.9358687
Rajan, S. P., \& Vivek, C. (2019). Analysis and Design of Microstrip Patch Antenna for Radar. Journal of Electrical Engineering \& Technology 14, 923-929.

Sağıroğlu, Ş., Güney, K., \& Erler, M. (1998). Resonant Frequency Calculation for Circular Microstrip Antennas Using Artificial Neural Networks. International Journal of RF and Microwave Computer-Aided Engineering, 270-277.

Sainati, R. A. (1996). CAD of Microstrip Antennas for Wireless Applications Nowood, MA: Artech House. Norwood: Artech House.

Sanchez, J. R. (2004). EM-Based Optimization Of Microwave Circuits Using Artificial Neural Networks: The State-OfThe-Art. IEEE Transactions on Microwave Theory and Techniques, 420-435.

Singh, V., Mishra, B., Tripathi, P. N., \& Singh, R. (2016). A Compact Quad-Band Microstrip Antenna For S And C-Band Applications. Microwave and Optical Technology Letters, 58(6), 1365-1369.

Tang, C. L., Chen, H., \& Wong, K. L. (1997). Small Circular Microstrip Antenna With Dual-Frequency Operation. Electronics Letters.

Türker, N., Güneş, F., \& Yıldırım, T. (2007). Artificial Neural Design of Microstrip Antennas. Turkish Journal of Electrical Engineering \& Computer Sciences, 445-453.

Zhang, W., Feng, F., Liu, W., Yan, S., Zhang, J., Jin, J., \& Zhang, Q.-J. (2021). Advanced Parallel Space-MappingBased Multiphysics Optimization for High-Power Microwave Filters. IEEE Transactions on Microwave Theory and Techniques vol.69, 2470-2484. 